\title{
Low immunogenicity of endothelial derivatives from rat embryonic stem cell-like cells
}

Juliane Ladhoff ${ }^{1}$, Michael Bader ${ }^{2}$, Sabine Brösel ${ }^{1}$, Elke Effenberger ${ }^{1}$, Dirk Westermann ${ }^{3}$, Hans-Dieter Volk ${ }^{1,4}$, Martina Seifert ${ }^{1,4}$

${ }^{1}$ Institute of Medical Immunology, Charité Universitätsmedizin Berlin, Campus Charité Mitte, Monbijoustrasse 2a, 10117 Berlin, Germany; ${ }^{2}$ Max-Delbrück-Center for Molecular Medicine, 13092 Berlin, Germany; ${ }^{3}$ Department of Cardiology and Pneumology, Charité Universitätsmedizin Berlin, Campus Benjamin Franklin Hospital, 12200 Berlin, Germany; ${ }^{4}$ Berlin-Brandenburg Center for Regenerative Therapies (BCRT), Charité Universitätsmedizin Berlin, Campus Virchowklinikum, 13353 Berlin, Germany

Embryonic stem cells (ESC) are suggested to be immune-privileged, but they carry the risk of uncontrolled expansion and malignancy. Upon differentiation they lose their tumor-forming capacity, but they become immunogenic by the expression of a normal set of MHC molecules. This immunogenicity might trigger rejection after application in regenerative therapies. In this study MHC expression of and immune responses to endothelial derivatives of rat embryonic stem cell-like cells (RESC) under inflammatory conditions were determined in comparison to primary rat aortic endothelial cells (ECs). Cellular as well as humoral allo-recognition was analyzed in vitro. In addition, immune reactions in vivo were assessed by allo-antibody production and determination of interferon- $\gamma$ (IFN $\gamma$ )-secreting allo-reactive T cells. RESC derivatives expressed low but significant levels of MHC class I, and no MHC class II. In response to IFN $\gamma$ MHC class I expression was enhanced, while class II transactivator induction failed completely in these cells; MHC class II expression remained consistently absent. Functionally, the RESC derivatives showed a reduced allo-stimulatory capacity, protection against humoral allo-recognition in vitro and a slightly diminished susceptibility to cytotoxic $\mathbf{T}$ cell lysis. Furthermore, in vivo experiments demonstrated that these cells do not trigger host immune reactions, characterized by no allo-antibody production and no induction of allo-reactive memory $T$ cells. Our results show that endothelial derivatives of RESC have a distinctive reduced immunogenic potency even under inflammatory conditions.

Keywords: stem cells, cell differentiation, endothelial cells, MHC, CIITA, immunogenicity

Cell Research (2009) 19:507-518. doi: 10.1038/cr.2009.21; published online 24 February 2009

\section{Introduction}

Embryonic stem cells (ESC) are considered to be a powerful tool in regenerative and transplantation medicine, but their immunogenic potential and behavior under transplantation conditions still remain a concern. There are several reports of a robust immune-privileged ('tolerogenic') state of ESC of mice, humans and rats after engraftment [1-3], while others described an increase of immunogenicity following transplantation with pro-

\footnotetext{
Correspondence: Martina Seifert

Tel: +49-30-450524198; Fax: +49-30-450524907

E-mail: martina.seifert@charite.de

Received 14 February 2008; revised 17 July 2008; accepted 6 October 2008; published online 24 February 2009
}

ceeding differentiation $[4,5]$. Expression of structures recognized by the host immune system varies between the species, e.g. murine ESC lack completely MHC class I on the cell surface, whereas ESC from human and rat display low expression levels of this molecule. MHC class II and costimulatory molecules are generally not expressed by ESC. Another 'tolerogenic' feature of these cells is the profound expression of Fas ligand (FasL) that would induce T-cell apoptosis via Fas-FasL interaction [1, $3]$. The major obstacle following transplantation is the recognition of donor antigens on the graft by the recipient's immune cells. This process is mediated by host $\mathrm{T}$ cells identifying several foreign surface molecules, such as MHC class I and class II, on the transplant. MHC molecules on the donor cell are able to activate recipient $\mathrm{T}$ cells directly (direct allo-recognition), particularly 
in the case of pre-established memory. Furthermore, uptake of shedded donor MHC by recipient APC initiates priming of host CD4 $\mathrm{T}$ cells (indirect allo-recognition), which in turn may provide help to activate allo-antibodyproducing $\mathrm{B}$ cells and to induce CD8 T-cell activation $[6,7]$. Under inflammatory conditions MHC molecules are upregulated by pro-inflammatory cytokines, such as interferon- $\gamma$ (IFN $\gamma$ ). Endothelial cells (ECs) express MHC class I and, in contrast to the human system, rat ECs do not express MHC class II constitutively [8, 9]. But as non-professional APC they are able to increase their MHC class I and to induce MHC class II expression in response to IFN $\gamma$, thereby inducing direct allorecognition [10-12]. The MHC class II level has a major impact on the immunogenic potential of ECs, which is of great interest particularly in a transplantation setting [13].

In this study we generated endothelial derivatives from the recently described rat embryonic stem cell-like cell (RESC) line C12. This Wistar Kyoto (RT1.') rat blastocyst-derived cell line with a proportion of about $70 \%$ euploid cells grows in a leukemia inhibitory factor (LIF)dependent manner in three-dimensional clumps showing the typical embryonic markers such as alkaline phosphatase and the stage-specific embryonic antigen-1 (SSEA1) $[1,14]$. It shares in this regard characteristic properties with other described rat ES cell lines [15]. In contrast to known ESC of murine origin [16], SSEA-1 was expressed in the ES cell-like cell line $\mathrm{C} 12$ in a mosaic-like pattern and the immunostaining for Oct- 4 was negative, the reason to call them 'stem cell-like' [14]. The latter feature distinguishes the $\mathrm{C} 12$ cell line from rat ESC described by Vassilieva et al. [15]. Pluripotency, which was demonstrated so far for ESC from mice and chicken [17], was shown by in vitro differentiation of the $\mathrm{C} 12$ original cell line into neuronal, glial, endothelial and hepatic lineages [14]. We investigated the obtained endothelial derivatives in comparison to adult primary rat aortic ECs. ECs are of special interest in the context of small-diameter artificial vascular grafts for reconstitution of coronary circulation and peripheral vasculature. As these grafts need to be endothelialized in vitro prior to transplantation to prevent thrombosis [18], ECs play a pivotal role in allorecognition since they are able to trigger rejection due to expression of MHC class I and class II on the surface of the cellular graft $[19,20]$.

We elucidated for the first time the responsiveness of the two different cell types to the pro-inflammatory cytokine IFN $\gamma$, which is of particular relevance after transplantation. Both cell populations upregulated MHC class I following cytokine treatment. In contrast, RESCderived cells were unable to induce MHC class II after IFN $\gamma$ stimulation, whereas class II upregulation in adult
ECs was substantial. We could reveal discrepancies in the IFN $\gamma$ signaling pathway in the RESC endothelial derivatives compared to their adult primary counterparts. Additionally, we gained further insight into the resulting functional characteristics of the RESC derivatives in vitro, namely a reduced allo-stimulatory capacity, a slightly diminished susceptibility to cytotoxic T-cell lysis and a protection against humoral allo-recognition. Furthermore, we could also show in first in vivo experiments that these cells do not trigger immune reactions in the host. Thus, these endothelial derivatives of RESC seem to have a distinctive reduced immunogenic potential even under inflammatory conditions.

\section{Results}

\section{Characteristics of the RESC derivatives}

The original RESC of the C12 line were driven into the endothelial lineage by withdrawal of LIF and culture on Matrigel-coated dishes, followed by further flow cytometric enrichment of vascular/endothelial (VE)Cadherin $(+)$ cells and final culture on $0.1 \%$ gelatine coating. The derived cells were characterized for their phenotype and compared to the original cell line and to adult primary ECs. Surface expression of several endothelial markers, such as MHC class I (RT1.A), MHC class II (RT1.B), platelet/EC adhesion molecule-1 (PECAM-1), intercellular adhesion molecule-1 (ICAM-1), vascular cell adhesion molecule-1 (VCAM-1) and VE-Cadherin, was determined by FACS analysis (Figure 1A). The original cell line as well as RESC derivatives constitutively displayed significant but relatively low levels of MHC class I, whereas adult ECs showed higher densities of MHC class I on the cell surface. However, MHC class II was undetectable in all cell types. The endothelial marker PECAM-1 was expressed in the original cell line while expression was diminished in the derivatives, but adult ECs displayed the highest surface densities of PECAM-1. Concerning the adhesion molecules, ICAM-1 levels were slightly increased in the RESC-derived cells compared to adult ECs and the cell line of origin. On the other hand VCAM-1, a marker for activated endothelium, was completely absent in the original cell line as well as in the derivatives, but present at a low degree in ECs. VE-Cadherin was expressed at high intensity in adult ECs. A small, but distinct, subpopulation of RESC derivatives displayed very high levels of this marker, while the majority showed a moderate VE-Cadherin staining. In the cell line of origin very few cells $(<2 \%)$ expressed this endothelial marker. Supplementary mRNA expression studies by quantitative real-time polymerase chain reaction (qRT-PCR) revealed the capacity of RESC 
derivatives to express mRNA of thrombomodulin, the endothelial specific thrombin receptor, to a similar extent as adult ECs (data not shown). In addition, we checked the morphological appearance of the RESC derivatives in comparison to their adult primary counterpart and the original cell line by light microscopy. We assessed that the RESC-derived cells displayed a cobble-stone like morphology (Figure 1Bb) as it is typical of adult ECs
(Figure 1Ba); however, the cell line of origin grew as partially adherent cells and formed clumps and clusters (Figure $1 \mathrm{Bc}$ ). Another feature of primary ECs is the expression of von Willebrand factor (vWF) protein in the cytoplasm (Figure 1Bd). For the RESC derivatives we detected a clear, characteristic intracellular staining by the anti-vWF antibody (Figure $1 \mathrm{Be}$ ), but in the cell line of origin vWF was completely absent (Figure

A
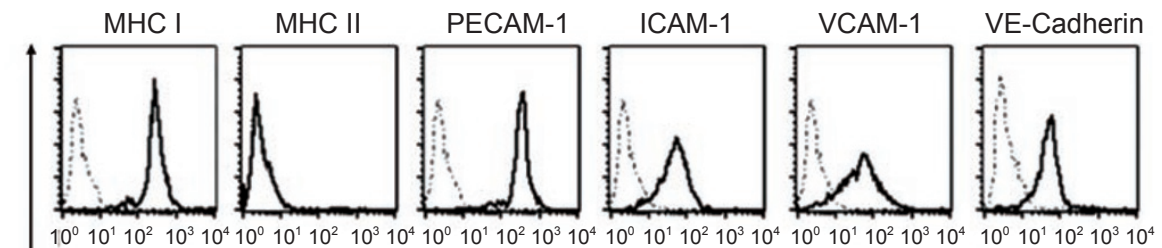

RESC

endothelial

derivatives
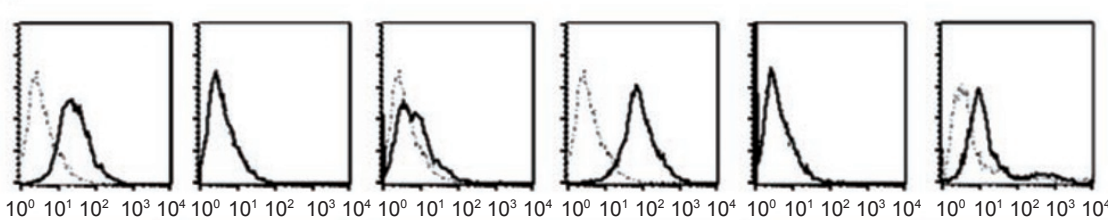

RESC line
of origin
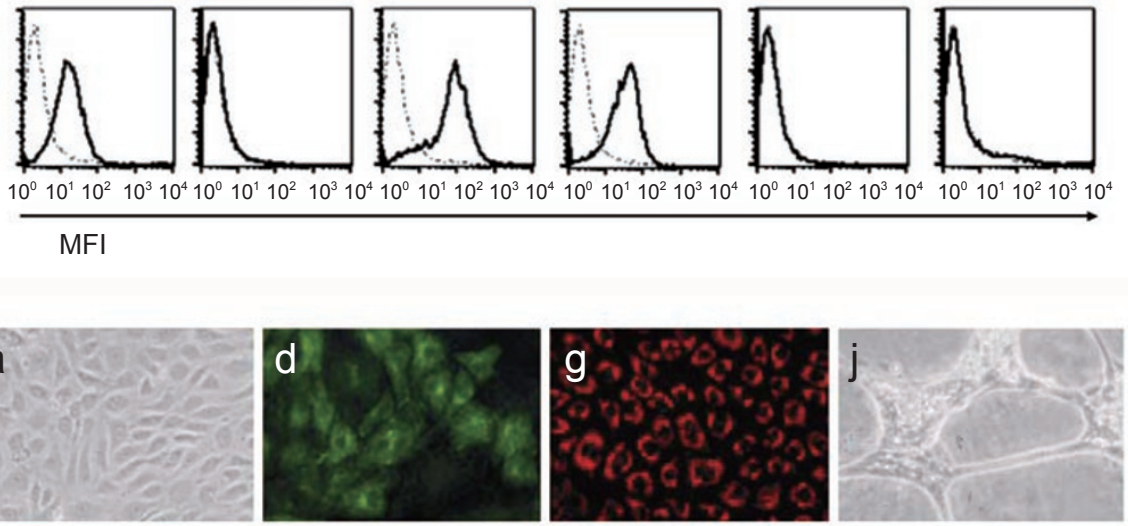

RESC

endothelial derivatives
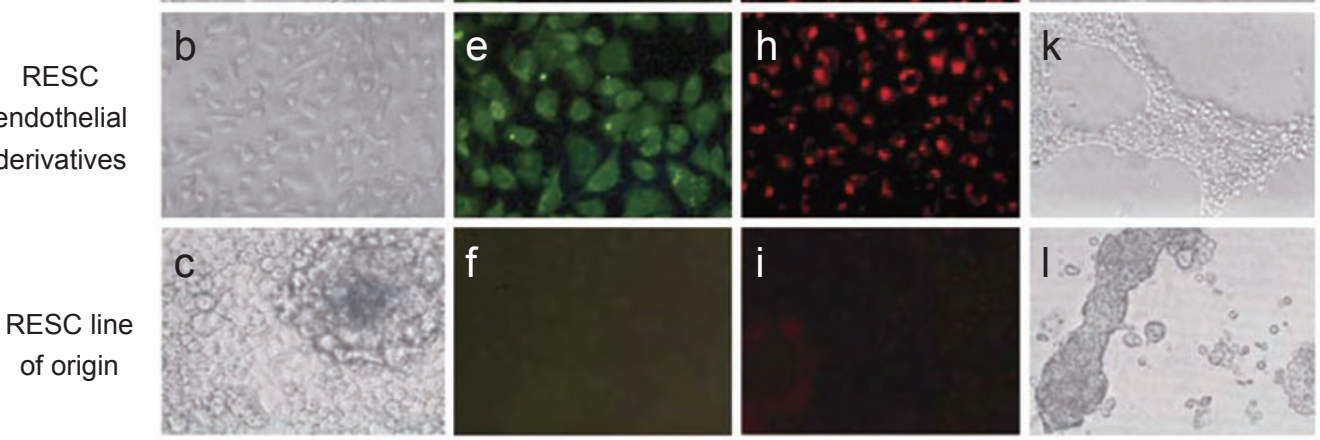

Figure 1 Phenotypical characteristics of the analyzed cell types. (A) Endothelial surface markers on adult ECs (upper row), RESC endothelial derivatives (middle row) and the cell line of origin (lower row). Expression levels of MHC class I, MHC class II, PECAM-1, ICAM-1, VCAM-1 and VE-Cadherin were analyzed flow cytometrically. Markers are shown (solid line) compared to an isotype identical control (dashed line) in representative histograms $(n=6)$. (B) Cobble-stone-like morphology of adult ECs (a), RESC derivatives (b), cluster formation of the cell line of origin (c); intracellular vWF expression (FITC staining) in ECs (d) and in RESC derivatives (e), no expression in the original cell line (f), Dil-Ac-LDL uptake (red-stained vesicles) by ECs (g) and RESC-derived cells (h), no uptake by the original cell line (i), tube formation of ECs (j) and RESC derivatives (k) and aggregation in clusters of the cell line of origin (I) on Matrigel after $24 \mathrm{~h}$ (original magnification $\times 200$ ). Representative images from three independent experiments are shown. 
1Bf). Furthermore, we investigated the ability of RESC derivatives to actively take up low-density lipoprotein (LDL), a typical characteristic of the endothelium. We could confirm by fluorescence microscopy that RESCderived cells as well as adult ECs were equally capable of taking up the modified lipoprotein Dil-Ac-LDL (Figure $1 \mathrm{Bg}$ and $1 \mathrm{Bh}$ ). On the other hand, only background fluorescence was detectable in the original cell line (Figure 1Bi). When cultured on Matrigel, an extracellular matrix analogue, the entirety of seeded RESC-derived cells assembled in branched complex tube-like structures within $24 \mathrm{~h}$ similar to adult ECs (Figure $1 \mathrm{Bj}$ and $1 \mathrm{Bk}$ ). In contrast, the original cells were unable to form those tubular vascular structures (Figure 1Bl). As the RESC derivatives exhibited several endothelial features, they will be called endothelial derivatives in the rest of the paper.

\section{Response to the pro-inflammatory cytokine IFN $\gamma$}

To study the responsiveness of the RESC derivatives to pro-inflammatory stimuli compared to adult ECs, cells were stimulated with $10 \mathrm{ng} / \mathrm{ml} \mathrm{IFN} \gamma$ for $24 \mathrm{~h}$ and surface expression of MHC class I and II was monitored. In both cell types a pronounced induction of MHC class I was detected after IFN $\gamma$ treatment demonstrating normal cytokine responsiveness. The extent of upregulation was comparable in both cell types. However, due to the different basal MHC class I expression levels, its surface density after cytokine treatment was significantly higher in adult ECs in comparison to the RESC-derived cells $(P=0.001)$. With regard to MHC class II expression, a substantial increase was detectable in adult ECs, but no induction was observed in RESC endothelial derivatives
$(P=0.003)$ (Figure 2). Titration experiments with IFN $\gamma$ in relation to $\mathrm{MHC}$ expression demonstrated a clear dose dependency concerning MHC class I induction, and a complete unresponsiveness of the RESC-derived cells concerning MHC class II expression at all cytokine concentrations (data not shown).

\section{Differences in IFN signal transduction}

The varying responses of the analyzed cell types to IFN $\gamma$ stimulation may point at discrepancies in the signal transduction pathway of this cytokine within these cells. Therefore, we investigated the IFN $\gamma$ receptor mRNA profile by qRT-PCR. Both receptor chains, IFN $\gamma R 1$ and IFN $\gamma$ R2, showed similar expression levels in adult ECs and RESC endothelial derivatives, independent of an IFN $\gamma$ treatment (Figure 3A). To examine the intracellular signaling, phosphorylation of the signal transducer and activator of transcription 1 (STAT1) was analyzed by flow cytometry. A strong increase of phosphorylated STAT1 (pSTAT1) was detected in adult ECs following treatment with $10 \mathrm{ng} / \mathrm{ml} \mathrm{IFN \gamma}$ for $5 \mathrm{~min}$. Induction of pSTAT1 was less pronounced in RESC endothelial derivatives (Figure 3B). To assay the transcriptional regulation of the IFN $\gamma$-induced MHC class II gene, expression of the class II transactivator (CIITA) was determined by qRT-PCR after $6 \mathrm{~h}$ of stimulation with $10 \mathrm{ng} / \mathrm{ml} \mathrm{IFN} \gamma$. The time point of analysis was selected according to kinetic studies indicating that CIITA mRNA expression in ECs reached a plateau after $6 \mathrm{~h}$ of IFN $\gamma$ treatment (data not shown). A substantial induction of CIITA mRNA by IFN $\gamma$ was detected in adult ECs, but in RESC derivatives expression stayed at basal levels (Figure 3C).
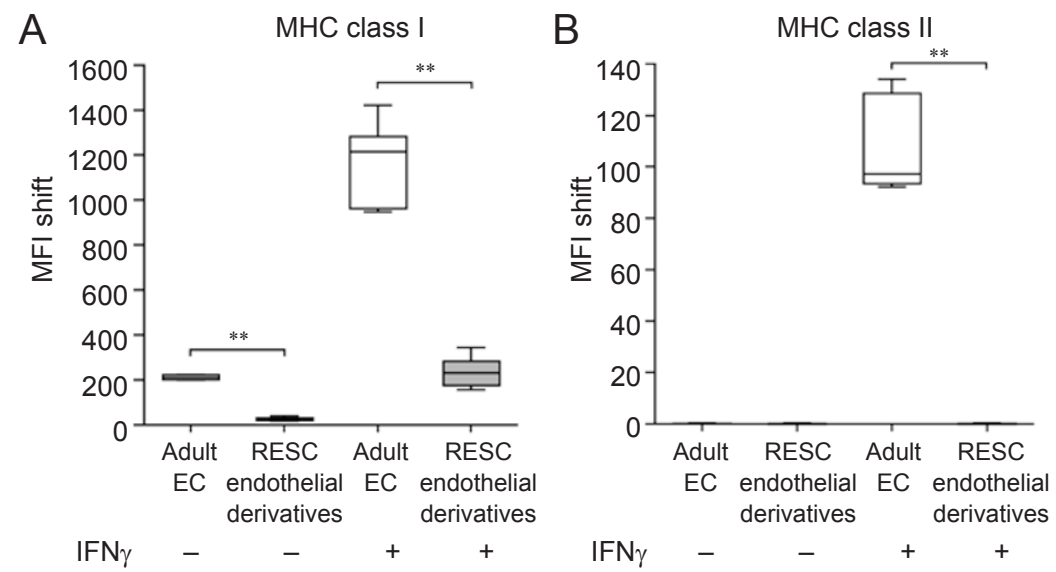

Figure 2 Induction of MHC surface expression following treatment with $10 \mathrm{ng} / \mathrm{ml}$ IFN $\gamma$ for $24 \mathrm{~h}$. (A) Basal levels as well as IFN $\gamma$-induced expression levels of MHC class I were significantly diminished in RESC endothelial derivatives compared to adult ECs. Data are presented as MFI shift \pm SEM of independent experiments $(n=6), * * P<0.01$. (B) No constitutive expression of MHC class II was detectable in both cell types. Following IFN $\gamma$ stimulation MHC II was substantially induced in adult ECs, but not in RESC derivatives. Data are presented as MFI shift \pm SEM of independent experiments $(n=6)$, *** $P<0.01$. 

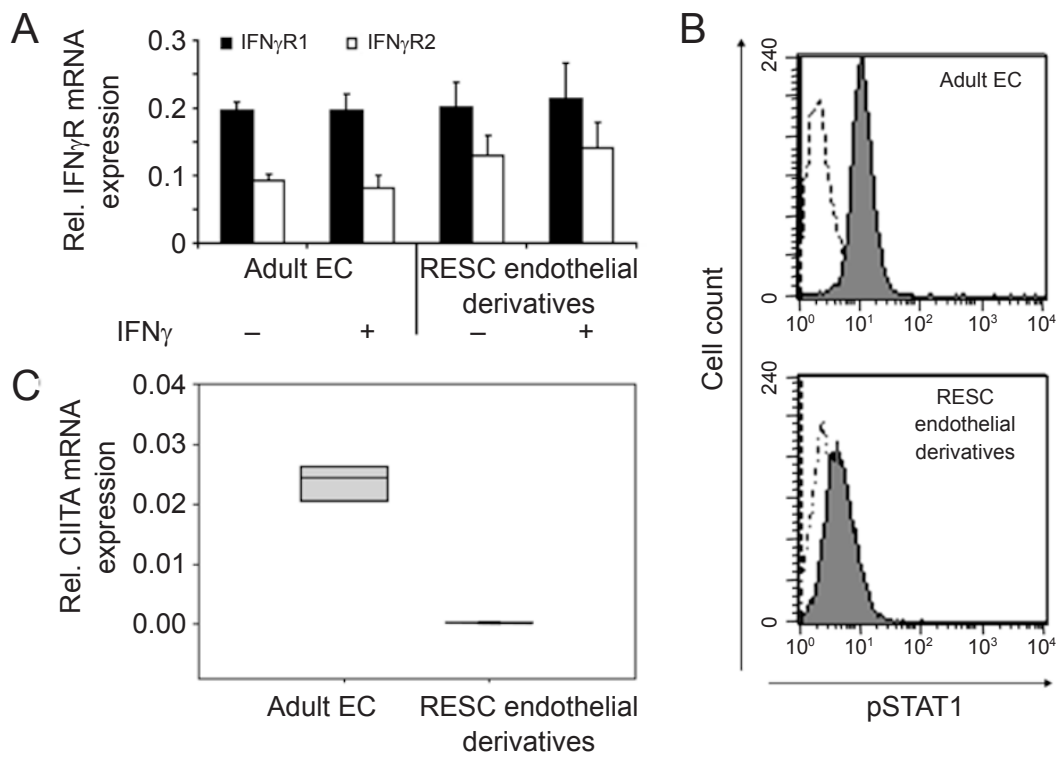

Figure 3 Impaired IFN $\gamma$-related signal transduction in RESC derivatives. (A) mRNA levels of IFN $\gamma$ receptor chain 1 (IFN $\gamma$ R1 - black bars) as well as IFN $\gamma$ receptor chain 2 (IFN $\gamma R 2$ - white bars) were equal in adult ECs and RESC endothelial derivatives. Levels stayed unchanged after IFN $\gamma$ stimulation. Independent samples were analyzed by qRT-PCR and normalized to the housekeeping gene expression $(n=6)$. (B) Prominent phosphorylation of STAT1 (pSTAT1 - gray curve) in adult ECs, but only a weak effect in RESC endothelial derivatives after IFN $\gamma$ stimulation. Unstimulated samples were pSTAT1-negative (dashed curves). pSTAT1 was detected intracellularly by FACS analysis after treatment with $10 \mathrm{ng} / \mathrm{ml}$ IFN $\gamma$ for $5 \mathrm{~min}$. One representative experiment is shown $(n=3)$. (C) mRNA expression of the class II transactivator (CIITA) following treatment with $10 \mathrm{ng} / \mathrm{ml}$ IFN $\gamma$ for $6 \mathrm{~h}$ was substantially induced in adult ECs, whereas remained unchanged in RESC-derived cells at the detection limit. Analysis was performed by qRT-PCR. All data are presented as mean \pm SEM of independent experiments $(n$ $=6)$.

RESC derivatives show low allo-stimulatory capacity in vitro

As MHC class II induction on ECs after cytokine stimulation is the key mechanism triggering allorecognition by $\mathrm{CD} 4(+) \mathrm{T}$ cells, we studied the allostimulatory capacity of both cell types in a CFDA-SEbased proliferation assay. Adult ECs as well as RESC derivatives were pre-incubated for $48 \mathrm{~h}$ with $10 \mathrm{ng} / \mathrm{ml}$ IFN $\gamma$ to induce MHC class II expression; control cells were left untreated and all samples were co-cultured with allogeneic CD4(+) responder cells. Strong proliferation of responder cells $(11 \%)$ already on day 3 revealed a high allo-stimulatory capacity of IFN $\gamma$-treated adult ECs. Almost no proliferation ( $2 \%$ ) was detected in co-cultures with IFN $\gamma$-pretreated RESC endothelial derivatives; similarly, minimal levels of allogeneic CD4(+) T cell proliferation were induced by unstimulated adult ECs (2\%) and RESC derivatives (1\%) (Figure 4A). T cell proliferation upon allo-stimulation by unstimulated cells and IFN $\gamma$-treated RESC endothelial derivatives was as low as auto-proliferation of the responder CD4(+) T cells and less than in co-cultures with IFN $\gamma$-stimulated syngeneic ECs. In contrast, allogeneic IFN $\gamma$-pretreated adult ECs induced significantly higher (12-fold) levels of CD4(+) T cell proliferation $(P=0.009)$ (Figure 4B).

Protection of RESC endothelial derivatives against allorecognition in vitro

Allo-antibody response represents a key limitation in the survival of allogeneic ECs after transplantation. Therefore, RESC derivatives were analyzed for their behavior against humoral immune attack compared to adult ECs. Pre-incubation of adult ECs with allo-specific serum followed by addition of complement resulted in substantial cell death. The serum-induced lysis was significantly (by $\sim 18$-fold) enhanced at 1:5 dilution compared to the spontaneous lysis without addition of serum $(P=$ 0.004 ) and decreased dose-dependently (Figure 5A). In contrast, RESC endothelial derivatives were completely resistant against complement-mediated lysis, independent of the serum concentrations; and cell lysis did not exceed the spontaneous level.

Furthermore, the susceptibility to cytotoxic T lymphocyte (CTL) lysis was analyzed in an assay using pre-activated allo-specific CTL. RESC endothelial derivatives exhibited marginally, but not significantly, diminished 
A

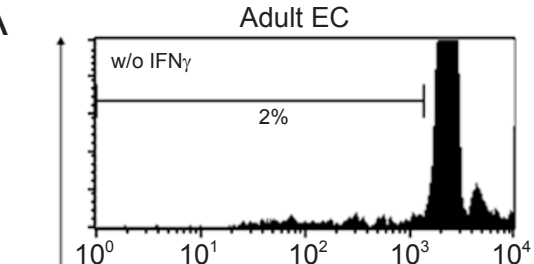

RESC endothelial derivatives

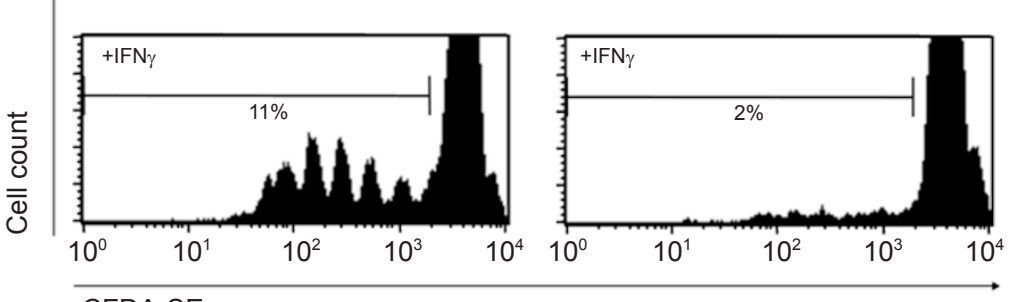

CFDA-SE

B

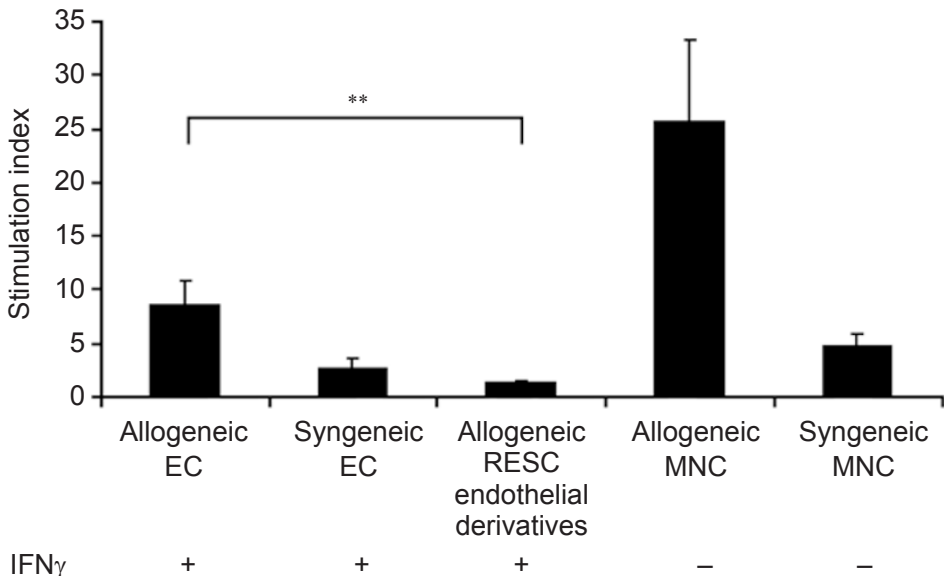

Figure 4 Low allo-stimulatory capacity of RESC endothelial derivatives. (A) Proliferation of allogeneic CD4(+) responder T cells in co-cultures with unstimulated and IFN $\gamma$-pretreated adult ECs and RESC derivatives on day 3 was analyzed by CFDASE-based proliferation assay. Representative histograms of five independent experiments are shown. (B) The allo-stimulatory capacity of IFN $\gamma$-pretreated adult ECs as well as RESC endothelial derivatives was determined in one-way MLRs with allogeneic CD4(+) responder T cells. Allogeneic ECs displayed a 10-fold higher stimulation index than allogeneic RESC derivatives. Their potential to trigger T cell proliferation was as small as that of syngeneic ECs and below that of syngeneic MNC. Stimulation indices were calculated as a quotient of the percentage of sample proliferation and the percentage of auto-proliferation of CD4(+) T cells without stimulus. Data are presented as mean \pm SEM of independent experiments $(n=5)$.

killing by allo-reactive effector T cells. Approximately $50 \%$ of these cells were killed by allo-specific CTL at an effector to target cell ratio of 100:1, whereas adult ECs displayed a maximum lysis rate of $65 \%$ under such conditions (Figure 5B). To determine non-specific killing generated by the cytotoxic $\mathrm{T}$ cells, adult ECs isolated from Wistar-Furth rats were utilized as the third party control, which possess a different MHC type (haplotype RT1 $\left.{ }^{u}\right)$. Respective co-incubation resulted in background lysis rates of around $10 \%$.

Low immunogenicity of RESC endothelial derivatives in vivo

Immunogenicity of RESC endothelial derivatives was analyzed in vivo by immunization experiments. Adult ECs as well as RESC-derived cells were left untreated or stimulated with $10 \mathrm{ng} / \mathrm{ml} \mathrm{IFN} \gamma$ for $72 \mathrm{~h}$ prior to i.p. injection into BDIX recipient rats. Humoral immune response was assayed by detection of circulating allo-specific IgG in recipients' sera 14 days and 28 days after challenge. Already at day 14 the induction of allo-IgG was prominent in animals receiving allogeneic IFN $\gamma$-stimulated adult ECs, with levels comparable to those of the positive control group receiving allogeneic splenocytes. However, injection of allogeneic IFN $\gamma$-pretreated RESC endothelial derivatives resulted in a significantly reduced allo-IgG response in comparison to the group that received IFN $\gamma$-treated adult ECs $(P=0.008)$. This response 
A

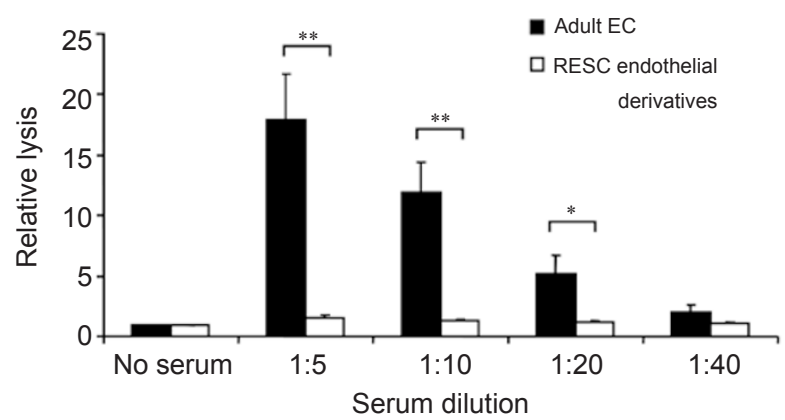

$\mathrm{B}$

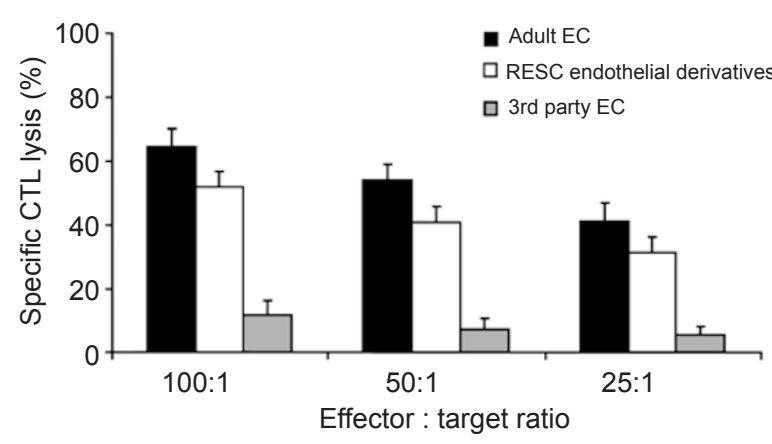

Figure 5 Reduced immune responses in vitro. (A) After sequential preincubation with allo-specific serum and complement, lysis was determined flow cytometrically by $7-A A D$ staining. Adult ECs exhibited an 18-fold higher lysis rate compared to spontaneous lysis without serum incubation at 1:5 dilution; RESC derivatives stayed at spontaneous lysis levels. Data are presented as mean \pm SEM of independent experiments $(n=6)$, ** $P<0.01$; $* P<0.05$. (B) Calcein-labeled adult ECs and RESC derivatives were incubated with allo-specific CTLs; specific lysis rates were determined spectrofluorometrically. Allogeneic ECs (black bars) were effectively killed by allo-specific CTL; in RESC derivatives lysis was marginally diminished (white bars). Non-specific killing was determined using adult $3^{\text {rd }}$ party ECs (gray bars). Data are presented as mean \pm SEM of independent experiments $(n=6)$.

was comparable to the negative control group, receiving PBS alone, as well as to the two groups injected with unstimulated adult ECs and RESC derivatives, respectively (Figure 6A). A similar allo-antibody profile was observed 28 days post immunization (data not shown). To analyze cellular immune reactions, allo-reactive IFN $\gamma$-producing $\mathrm{T}$ cell frequencies were determined in recipient splenocytes 28 days after immunization by allogeneic restimulation. ELISPOT assay of these cells revealed a moderate, but distinct frequency of allo-reactive $\mathrm{T}$ cells in animals injected with IFN $\gamma$-pretreated adult ECs. In contrast, T-cell response in recipients of IFN $\gamma$-stimulated RESC endothelial derivatives did not exceed background levels of PBS-injected control animals. IFN $\gamma$-producing allo-reactive T-cell frequency was pronounced in the positive control group receiving allogeneic splenocytes
(Figure 6B).

\section{Discussion}

In this study we characterized the immunogenic features of the RESC C12 endothelial derivatives in comparison to the fully differentiated rat aortic EC counterpart and investigated their immune responses in vitro and in vivo. Our data showed that both the RESC C12 derivatives and rat aortic ECs expressed MHC class I, although at different expression levels, and lacked a constitutive MHC class II expression. The RESC derivatives displayed a subset of typical EC features, such as VE-
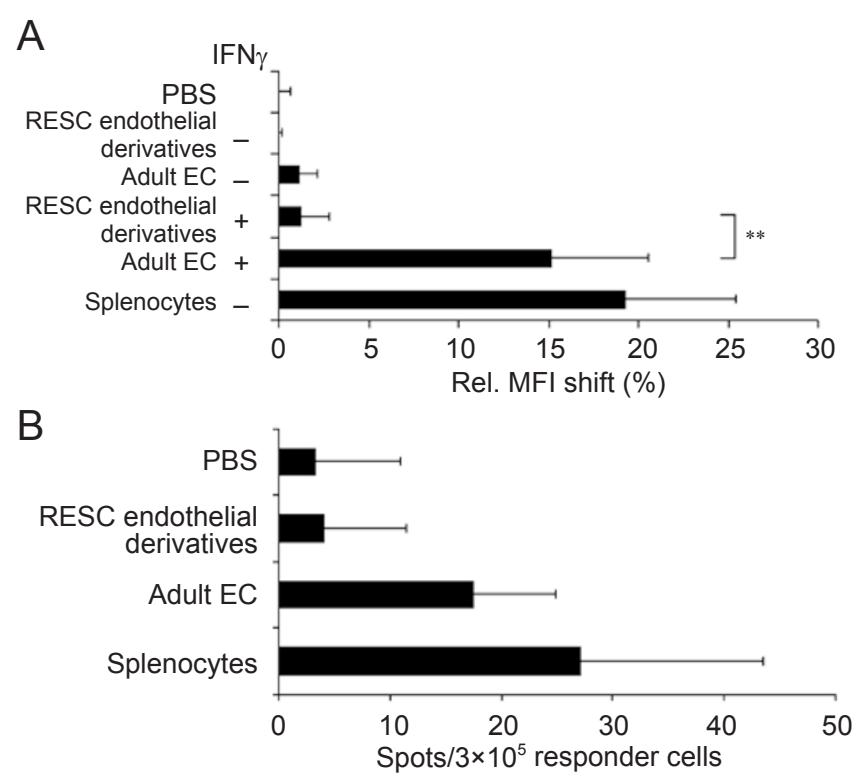

Figure 6 Lacking immune responses in vivo after allogeneic immunization. (A) IgG-allo-antibody serum levels 14 days after i.p. immunization with allogeneic splenocytes (positive control) and IFN $\gamma$-pretreated adult ECs were similar. IFN $\gamma$-pre-stimulated RESC derivatives induced minimal allo-antibody production as well as unstimulated adult ECs and RESC derivatives. Analysis was performed flow cytometrically by incubating fresh isolated thymocytes of the allogeneic stimulator rat strain with sera of the recipient rats followed by incubation with an anti-lgG-FITClabeled detection antibody. Data are shown as MFI shift (\%) relative to an internal positive standard control serum \pm SEM of independent experiments $(n=6),{ }^{*} * P<0.01$. Relative values were calculated as (experimental MFI shift $\times 100 \%$ )/MFI shift of the internal positive standard. (B) Allo-reactive IFN $\gamma$-producing memory $\mathrm{T}$ cells in recipient spleens were detected by IFN $\gamma$ ELISPOT assay 28 days after challenge. The frequency of IFN $\gamma$ producers was highest in rats receiving splenocytes, slightly diminished in recipients of IFN $\gamma$-pretreated adult ECs and reduced to background levels in animals immunized with IFN $\gamma$-stimulated RESC endothelial derivatives. Data are shown as mean \pm SEM of independent experiments $(n=6)$. 
Cadherin and intracellular vWF expression, LDL uptake and the ability to form tube-like structures on an extracellular matrix analogue in vitro. Moreover, in preliminary experiments we could verify the contribution to the formation of vascular structures in vivo by application of Dil-labeled RESC C12 derivatives into skin grafts or in a mouse model of hind limb ischemia (data not shown). In contrast, the undifferentiated RESC did not exhibit these endothelial key characteristics. The original cell line C12 was described to contain $70 \%$ of euploid cells and to express alkaline phosphatase uniformly and SSEA-1 in a mosaic-like pattern $[1,14]$. Pluripotency of the cells could be verified by differentiation into cells of all three germ layers. In contrast to mouse ES cell lines the immunostaining for the transcription factor Oct-4 was reported to be unsuccessful, the reason to call them stem cell-like cells [14]. In particular, our experimental data support and supplement the initial description of Ruhnke et al. [14] by a combined differentiation and sorting method based on VE-Cadherin expression that led to endothelial derivatives with a full set of endothelial markers. RESC C12 endothelial derivatives did not express FasL, a characteristic described for the cell line of origin [1]. Additionally, unlike the adult ECs, they were negative for the apoptosis-mediating molecule Fas (data not shown).

Differences in the expression level of individual EC markers between the RESC-derived cells and the aortic ECs might be attributed to that of fetal and adult largevessel tissues, whereas the fetal vessels may represent a microvascular phenotype. In the past differential expression of endothelial markers was described for the ECs of fetal and maternal vessels in the placenta, as well as for vascular trophoblasts [21]. The strong expression of ICAM-1 and absence of VCAM- 1 on placental endothelium resemble the features of our RESC endothelial derivatives.

Since the immunogenicity of ECs is strongly dependent on the inducible expression of MHC class II, we analyzed the two different cell types with regard to their capacity to express MHC class II after stimulation with IFN $\gamma$. Surprisingly, although both cell types upregulated MHC class I in response to a range of concentrations of IFN $\gamma$, MHC class II could be induced only in adult ECs, but not in the RESC-derived cells. This unresponsiveness with regard to MHC class II was associated with a missing CIITA expression in RESC derivatives. For the original cell line C12 MHC class II induction was reported in a small subpopulation after in vivo application, illustrating the general competence of these cells to express MHC class II [1].

Other cytokines that might play a role in the inflammatory response during transplantation, like TNF- $\alpha$ and
IL-1 $\beta$, could induce vascular adhesion molecules and upregulate the expression of the tissue factor [21]. However, both cytokines were unable to induce either MHC class I or MHC class II in ECs and RESC derivatives under our experimental conditions (data not shown).

Most importantly, in functional studies we were able to demonstrate a diminished induction of immune response in vivo after cell transplantation of IFN $\gamma$-treated RESC derivatives into an allogeneic recipient, while IFN $\gamma$-stimulated ECs induced a strong allo-response. Indeed, allo-antibody production and allo-reactive IFN $\gamma$ secreting memory $\mathrm{T}$ cells in spleens of rats immunized with RESC derivatives were hardly detectable. Moreover, RESC derivatives are also more resistant to allogeneic effector mechanisms. Compared to ECs, they were protected from allo-antibodies/complement-mediated responses and showed a marginally reduced susceptibility to the attack of allo-reactive CTL in vitro. In contrast to sterile rodents, this is of particular importance for the human situation. Patients show frequently allo-specific memory $\mathrm{T}$ or $\mathrm{B}$ cells even before transplantation due to cross-reactivity resulting from a life in a less pathogenfree environment.

It is widely accepted that allogeneic transplants initiate immunological rejection processes. Therefore we would assume that transplantation of allogeneic stem cell derivatives should follow these rules. However, there are different and inconsistent data about MHC class I and class II expression on mouse or human stem cells before and after differentiation. It was shown that human ES cells express MHC class I at low levels and that stimulation by IFN $\gamma$ upregulates the level of expression and induces MHC class II [22]. In vitro studies with human ES cells also demonstrated that cytotoxic immune responses by $\mathrm{T}$ cells were exclusively induced following IFN $\gamma$ stimulation and that natural killer (NK) cell response was negligible $[23,24]$. In some studies mouse ES cells exhibited a strong humoral and cellular immune response after in vivo application into injured myocardium in allogeneic settings [4, 5]. In contrast, Bonde et al. [3] described the inhibition of allo-responses to donor MHC antigens and the development of a multi-lineage mixed chimerism after in vivo administration of mouse ES cells into sublethally irradiated allogeneic hosts.

In our rat model we used a well-characterized RESC line (C12) [1] and were able to drive its differentiation into the endothelial lineage. Although the RESC C12 derivatives expressed low levels of MHC class I on the surface, we could show that allo-antibody together with complement failed to induce lysis of these cells in contrast to a strong effect on adult ECs. The low levels of MHC class I, however, are still sufficient to prevent 
complete resistance of the RESC derivatives to the killing by pre-activated allo-reactive CTL although the lysis was reduced compared to ECs. As these cells are not able to prime allo-reactive T cells in vivo (see above), it is unlikely that they are able to trigger CTL generation even in the presence of allo-reactive memory T cells.

The most prominent difference between the RESC endothelial derivatives and the adult and fully differentiated aortic ECs was the absence of MHC class II on the RESC derivatives even after stimulation with IFN $\gamma$. Although IFN $\gamma$ receptor chains R1 and R2 were equally expressed in the RESC-derived cells and adult ECs, the detected lower STAT1-phosphorylation in RESC C12derived cells was a strong hint for a defective signaling within the pathway of MHC class II induction. This was corroborated by the lack of CIITA expression at mRNA level in RESC-derived cells. A similar mechanism is known from trophoblasts, which are unable to induce MHC class II in response to IFN $\gamma$, caused by an epigenetic silencing of CIITA transcription [25].

The MHC class II expression on transplanted cells could affect their antigenic properties, their antigen-presenting capacity and also their features as a target for rejection effector's mechanisms. The impact of MHC class II expression on rat vascular ECs from the heart or their immunogenic potential in vivo was clearly demonstrated long ago and deficient MHC class II expression by disrupting the JAK/STAT pathway was indicated as a mechanism of human cytomegalovirus to prevent CD4(+) T cell-mediated immune responses $[13,26]$. CIITA in this regard is known as a master regulator for the transcription of all MHC class II genes and other components of the antigen-presenting pathway [27, 28]. In this way CIITA expression might increase the allogeneic response to an allograft.

Our proliferation studies with isolated CD4(+) CFDASE labeled T cells emphasize the low immunogenic state of the RESC-derived ECs due to their inability to upregulate MHC class II after stimulation. The allo-stimulatory capacity of the RESC derivatives lies in the same order of magnitude as the syngeneic control or even lower. In contrast, the allogeneic adult rat ECs show a marked stimulation index comparable to that of the positive control with allogeneic mononuclear cells (MNC). For cells of the endothelial differentiation lineage, it was shown that CIITA-induced MHC class II expression is able to trigger proliferation of $\mathrm{CD} 4(+) \mathrm{T}$ cells independent of other T-cell costimulatory ligands [29]. The importance of the inducible MHC class II expression for the immunogenicity of allogeneic adult mesenchymal stem cells (MSC) and their osteogeneic derivatives was discussed by Liu et al. [30]. In that study the differentiated MSC were also not able to express MHC class II after IFN $\gamma$ stimulation in vitro, but expression gradually occurred at later time points after transplantation in vivo. It could be speculated that derivatives of adult stem cells and those of ESC behave differently in terms of induction of MHC class II expression and the cognate intracellular signaling pathways.

In conclusion, we demonstrated that the low immunogenic features of endothelial derivatives of a RESC line in vitro and in vivo are mainly caused by the inability of these cells to upregulate MHC class II on their surface after IFN $\gamma$ or allo-stimulation. This allows an insight into possible strategies such as inhibition of CIITA expression to reduce the immunogenicity of grafted cells for regenerative therapies in the future.

Although this first study demonstrated the immuneprivileged status of the endothelial derivatives from embryonic origin, it is likely that other risks may arise after transplantation, e.g. possible degeneration through an increased proliferation rate in vivo, or potential NK cell effects on the grafted cells in the context of their low MHC class I expression.

\section{Materials and Methods}

\section{Cell culture}

Primary rat aortic ECs were isolated from Wistar-Kyoto (WKY; haplotype RT1 ${ }^{1}$ ) rats as described elsewhere [31]. ECs were cultured in EC basal medium (EBM) supplemented with $10 \%$ fetal calf serum (FCS) (both PAA, Pasching, A), $50 \mu \mathrm{g} / \mathrm{ml}$ gentamycin, $2 \mathrm{mM}$ L-glutamine and $6 \mu \mathrm{g} / \mathrm{ml}$ EC growth supplement (all Sigma, Steinheim, D). Tissue culture plastic dishes were coated with $0.2 \%$ gelatine (Sigma, Steinheim, D). The RESC line C12 (derived from WKY rats) was kindly provided by Prof. Michael Bader, MaxDelbrück-Center for Molecular Medicine, Berlin. Initially RESC C12 were cultivated on tissue culture dishes in DMEM (PAA, Pasching, A) supplemented with 15\% FCS (Biochrom AG, Berlin, D), $100 \mathrm{U} / \mathrm{ml}$ penicillin, $100 \mu \mathrm{g} / \mathrm{ml}$ streptomycin (Life Technologies, Karlsruhe, D), 1\% MEM nonessential amino acids (Gibco, Karlsruhe, D), nucleosides $(8 \mu \mathrm{g} / \mathrm{ml}$ adenosine, $8.5 \mu \mathrm{g} / \mathrm{ml}$ guanosine, $7.3 \mu \mathrm{g} / \mathrm{ml}$ cytosine, $7.3 \mathrm{~g} / \mathrm{ml}$ uridine, $2.4 \mu \mathrm{g} / \mathrm{ml}$ thymidine), 10 $\mathrm{mM} \beta$-mercaptoethanol (all Sigma, Steinheim, D), $1.7 \mathrm{nM}$ human recombinant insulin (Roche Diagnostics, Mannheim, D) and 1000 $\mathrm{U} / \mathrm{ml}$ leukemia inhibitory factor (LIF; Chemicon, Hampshire, UK). They grew in clumps and clusters with partial adherence. Cells were driven into the endothelial lineage by culture on Matrige ${ }^{\mathrm{TM}}$ of a concentration of $9 \mathrm{mg} / \mathrm{ml}$ (BD Biosciences Pharmingen, San Diego, USA), withdrawal of LIF and a following flow cytometric enrichment of VE-Cadherin $(+)$ cells. For further culture cells were kept in $0.1 \%$ gelatine-coated culture dishes. The medium was changed every 2-3 days. Cells were passaged and harvested by treatment with $0.05 \%$ Trypsin/0.2\% EDTA (w/v) (Life Technologies, Karlsruhe, D). To titer the IFN $\gamma$ concentration for stimulation, cells were incubated with $0.1,1,10,50$ and $100 \mathrm{ng} / \mathrm{ml} \mathrm{IFN} \gamma$ for $24 \mathrm{~h}$. For stimulation experiments cells were treated with $10 \mathrm{ng} / \mathrm{ml}$ recombinant rat IFN $\gamma$ (HbT, Uden, NL, kindly provided by Prof. 
Burmann) as indicated for $5 \mathrm{~min}, 6,24,48$ or $72 \mathrm{~h}$; unstimulated controls were cultured in medium alone for an equal time span.

\section{Flow cytometry}

Surface expression patterns as well as phosphorylation status were analyzed by flow cytometry. Surface markers were stained using the mouse-anti-rat mAb to RT1.A (OX-18), RT1.B (OX-6), PECAM-1 (TLD-3A12), ICAM-1 (1A29), VCAM-1 (MR106) and an isotype control $\operatorname{IgG}_{1}{ }_{\kappa} \mathrm{mAb}$ (MOPC-21) (all BD Biosciences Pharmingen, San Diego, USA). Further surface staining was prepared with a rabbit-anti-rat polyclonal antibody to VE-Cadherin (Alexis Biochemicals, Lausen, $\mathrm{CH}$ ) and an appropriate isotype control. As secondary antibodies a PE-labeled donkey-anti-mouse $\operatorname{IgG}(\mathrm{H}+\mathrm{L})$ polyclonal antibody (Dianova, Hamburg, D) and a PElabeled goat-anti-rabbit IgG $(\mathrm{H}+\mathrm{L})$ polyclonal antibody (Southern Biotechnology, Birmingham, USA) were used. Phosphorylation of STAT1 was determined using the BD ${ }^{\mathrm{TM}}$ Phosflow Kit including the PE-labeled anti-phospho-STAT1 (pY701) antibody (BD Biosciences Pharmingen, San Diego, USA) according to the manufacturer's instructions. Measurements were carried out by means of a FACSCalibur ${ }^{\mathbb{B}}$ flow cytometer (Becton Dickinson, San José, USA); for analysis the software CellQuest (Becton Dickinson) was used.

\section{LDL uptake}

ECs and RESC derivatives were cultured in 24-well plates until reaching $80-90 \%$ confluence. The medium was removed and

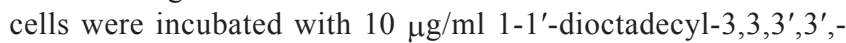
tetramethyl-indo-carboxyanine perchlorate-labeled LDL (Dil-AcLDL) (CellSystems, St Katharinen, D) for $4 \mathrm{~h}$ at $37{ }^{\circ} \mathrm{C}$. Subsequently, cells were washed twice with medium and fixed with $4 \%$ paraformaldehyde. Sample analysis was performed by fluorescence microscopy at $580 \mathrm{~nm}$.

\section{Intracellular $v W F$ staining}

ECs as well as RESC derivatives were cultured in 24-well plates until they reached $80-90 \%$ confluence. After fixation with $4 \%$ paraformaldehyde and permeabilization by $0.3 \%$ Triton X-100 (Serva, Heidelberg, D), cells were stained with a primary polyclonal rabbit-anti-human anti-vWF-antibody, which was crossreactive with rat. As secondary antibody, a polyclonal anti-rabbit IgG-FITC conjugate (both from Sigma, Steinheim, D) was used. For analysis samples were subjected to fluorescence microscopy at $488 \mathrm{~nm}$.

\section{Tube-forming assay}

ECs and RESC derivatives were seeded on Matrigel ${ }^{\mathrm{TM}}$ (BD Biosciences Pharmingen, San Diego, USA) of a concentration of $9 \mathrm{mg} / \mathrm{ml}$ with a cell density of $1 \times 10^{5} /$ well in $500 \mu \mathrm{L}$ medium in a 24-well plate and incubated up to $24 \mathrm{~h}$ at $37^{\circ} \mathrm{C}$. Tube formation was followed by light microscopy.

\section{Quantitative real-time polymerase chain reaction}

Total RNA was extracted from cultured cells using the Absolutely RNA RT-PCR Miniprep Kit (Stratagene, La Jolla, USA) according to the manufacturer's instructions. Isolated RNA in the measure of $2 \mu \mathrm{g}$ was reverse transcribed into cDNA using oligo dT-primers to be subjected to quantitative real-time PCR. Analyses were performed using the $\mathrm{SYBR}^{\circledR}$ Green PCR Kit (Applied
Biosystems, Foster City, USA) and primers designed for rat IFN $\gamma$ receptor chain 1 (IFN $\gamma \mathrm{R} 1)$, rat IFN $\gamma$ receptor chain $2(\mathrm{IFN} \gamma \mathrm{R} 2)$ and rat CIITA: sequences for IFN $\gamma R$ 1, forward 5'-AGT CGT CTT TCT GGC AAG TTA ATA CA-3' and reverse 5'-AAG CAG CAT CCA AAT TGA TTC TTC-3'; for IFN $\gamma$ R2, forward 5'-CAT CGC AGA GAC GAA ATG TGA-3' and reverse 5'-GCG CAG GAA GAC TGT GTA TGA GT-3'; for CIITA, forward 5'-CAA GGA CCT CTT CAT ACA GCA CAT T-3' and reverse 5'-GGA GGC ACT AGT TTC CTG TGC TT-3' (all synthesized by Metabion International AG, Martinsried, D). All primers were designed using Primer Express software (Applied Biosystems, Darmstadt, Germany). The amplification was performed using the 7500 Real-Time PCR System and analyzed with the 7500 System SDS Software (both from Applied Biosystems, Foster City, USA). Specificity of the desired gene products was documented by melting curve analysis. For normalization, expression of the housekeeping gene $\beta$-actin was used. Relative expression levels of the target gene mRNA were calculated by means of the formula $-2^{\Delta C t}$.

\section{Cytotoxicity assay}

ECs and RESC derivatives were labeled with the fluorescence dye Calcein-AM (20 $\mu \mathrm{M}$; MoBiTec, Göttingen, D) in serum-free medium for $30 \mathrm{~min}$ at $37^{\circ} \mathrm{C}$. Calcein-labeled target cells were cocultured at the given ratio with pre-activated allospecific cytotoxic T cells (CTL) in complete RPMI 1640 (PAA, Pasching, A) supplemented with $10 \% \mathrm{FCS}, 100 \mathrm{U} / \mathrm{ml}$ penicillin, $100 \mu \mathrm{g} / \mathrm{ml}$ streptomycin, $2 \mathrm{mM}$ L-glutamine, $5 \mathrm{mM}$ HEPES, and $50 \mu \mathrm{M} \beta$-mercaptoethanol for $4 \mathrm{~h}$ at $37{ }^{\circ} \mathrm{C}$. Alloantigen-specific CTL were generated in a 5-day mixed lymphocyte culture using lymph node-derived lymphocytes of Wistar-Kyoto (WKY, MHC haplotype RT1'; irradiated stimulator cells) and BDIX (MHC haplotype RT1 ${ }^{\mathrm{dv} 1}$; responder cells) rats. T cell-mediated lysis of the target cells was detected by Calcein release into the supernatant using a spectrofluorometer (TECAN, Crailsheim, D). Specific lysis was calculated in correlation to spontaneous (medium alone) and maximum $(0.9 \%$ Triton $\mathrm{X}-100)$ Calcein release by the target cells.

\section{Allo-antibody/complement assay}

ECs and RESC derivatives $\left(2 \times 10^{5}\right.$ cells/sample $)$ were pre-incubated with an allo-specific serum diluted in phosphate-buffered saline (PBS) supplemented with 2\% FCS as indicated for $30 \mathrm{~min}$ at RT. After washing off the allo-specific serum, samples were subsequently incubated in the presence of baby rabbit complement (Accurate Chemicals, Westbury, USA) 1:2 diluted in $\mathrm{GVB}^{++}$buffer (Sigma, Steinheim, D) for $1 \mathrm{~h}$ at $37^{\circ} \mathrm{C}$. Samples were washed and stained with the vital dye 7-actino-aminomycin D (7-AAD, Sigma, Steinheim, D) for $15 \mathrm{~min}$ at RT in the dark. 7-AAD staining was assessed immediately by flow cytometry. Relative lysis rates were calculated as quotient of specific lysis 7-AAD + cells with allospecific serum/spontaneous lysis 7-AAD+ cells without serum.

\section{One-way mixed lymphocyte reaction}

Stimulator cells were prepared as follows: allogeneic (WKY), syngeneic (BDIX) ECs and RESC endothelial derivatives were seeded in a 96-well plate and pretreated for $48 \mathrm{~h}$ with $10 \mathrm{ng} / \mathrm{ml}$ IFN $\gamma$ or left unstimulated for an equal time span. WKY and BDIX $\mathrm{MNC}$, serving as controls, were freshly isolated from lymph nodes and seeded in 96-well plates. All stimulator cells were irradiated for $15 \mathrm{~min}$ (30 Gray) and washed once subsequently. Responder 
CD4(+) T cells were isolated from BDIX lymph nodes by MACS separation using rat CD4-Microbeads (Miltenyi Biotec $\mathrm{GmbH}$, Bergisch-Gladbach, D). Purity of isolated CD4(+) T cells was assessed flow cytometrically by double staining with a FITC-labeled mouse anti-rat-CD3 $\mathrm{mAb}$ and a PE-labeled mouse anti-rat-CD4 $\mathrm{mAb}$ (both from BD Biosiences Pharmingen, San Diego, USA) and was found to range between $95-98 \%$. CD4(+) T cells were labeled with $2.5 \mu \mathrm{M}$ CFDA-SE (Molecular Probes, Karlsruhe, D) for $4 \mathrm{~min}$, followed by three washing steps with FCS-containing medium. CD4(+) cells were plated in a responder to stimulator ratio of 10:1 for adherent cells and a ratio of 1:1 for MNC and cultured in complete RPMI 1640 supplemented with $10 \%$ FCS, 100 $\mathrm{U} / \mathrm{ml}$ penicillin, $100 \mu \mathrm{g} / \mathrm{ml}$ streptomycin, $2 \mathrm{mM}$ L-glutamine, 5 mM HEPES, $50 \mu \mathrm{M} \beta$-mercaptoethanol and $2 \%$ autologous serum up to 5 days at $37^{\circ} \mathrm{C}$. To assess the auto-proliferation, CD4(+) responder cells were cultured in medium alone without any stimulation. Samples were collected on day 3 and day 5, and stained with a PE-labeled mouse anti-rat-CD4 mAb (BD Biosiences Pharmingen, San Diego, USA) for further FACS analysis with gating on the CD4(+)/CFDA-SE(+) lymphocyte population. Stimulation index was calculated as the quotient of sample proliferation and auto-proliferation of CD4(+) cells without stimulation.

\section{Immunization}

All animal experiments were approved by the local authorities for animal experiments. For immunization experiments adult ECs and RESC endothelial derivatives were pretreated with $10 \mathrm{ng} / \mathrm{ml}$ IFN $\gamma$ for $72 \mathrm{~h}$ at $37^{\circ} \mathrm{C}$ or left untreated. Splenocytes were freshly isolated from WKY rats. Naïve BDIX recipient rats (haplotype RT1 ${ }^{\text {dv1 }}$; Charles River Laboratories, Sulzfeld, D) were injected i.p. with a total of $3 \times 10^{6}$ cells in $300 \mu \mathrm{PBS}$ and PBS alone, respectively.

\section{Allo-antibody detection}

Sera of recipients were collected at day 14 and day 28 after immunization to determine the occurrence of circulating alloantibodies. First $2 \times 10^{5}$ freshly isolated BDIX thymocytes were incubated with 1:10, 1:50 and 1:100 dilutions of the recipient sera for $45 \mathrm{~min}$ at $4{ }^{\circ} \mathrm{C}$. After washing, samples were incubated with FITC-conjugated anti-rat-IgG mAb (AbD Serotec, Düsseldorf, D) for $30 \mathrm{~min}$ at $4{ }^{\circ} \mathrm{C}$. A serum from a BDIX rat transplanted with a WKY skin graft in the same dilution was used as internal standard and positive control; serum from a naïve BDIX rat served as negative control. Samples were analyzed on a FACSCalibur ${ }^{\circledR}$ flow cytometer (Becton Dickinson, San José, USA); for analysis the software CellQuest ${ }^{\mathbb{B}}$ (Becton Dickinson) was used. As strong variances between the individual fresh thymocyte preparations were observed (range of the mean fluorescence intensity (MFI) shift vs. the negative control between 21 and 91), relative values of the experimental MFI shift were assessed to exclude the inter-individual variations. Relative values were calculated as (experimental MFI shift $\times 100 \%$ )/MFI shift of the internal standard.

\section{Detection of allo-reactive IFN $\gamma$-producing $T$ cells}

Spleens from each recipient were harvested at day 28 after immunization to assess the number of allo-reactive IFN $\gamma$-secreting $\mathrm{T}$ cells by rat IFN $\gamma$-ELISPOT according to the manufacturer's instructions (Diaclone, Besançon, F). MNC were isolated from spleens by density gradient centrifugation using Pancoll rat (PAN-
Biotech GmbH, Aidenbach, D). Splenocytes of BDIX recipients $\left(3 \times 10^{5} /\right.$ well $)$ were stimulated with RPMI 1640 medium alone (negative control), $2 \mathrm{ng} / \mathrm{ml}$ PMA and $2 \mathrm{pg} / \mathrm{ml}$ ionomycin (both Sigma, Steinheim, D) (positive control) or $3 \times 10^{5}$ stimulator cells (WKY splenocytes) in RPMI 1640 medium for $20 \mathrm{~h}$ at $37{ }^{\circ} \mathrm{C}$. After three washing steps with PBS containing $0.1 \%$ Tween 20 (Roth $\mathrm{GmbH}$, Karlsruhe, D) a biotinylated anti-rat IFN $\gamma$ detection antibody was added for $3 \mathrm{~h}$. After a final $45 \mathrm{~min}$ incubation period with a streptavidin-alkaline phosphatase conjugate and subsequent administration of the staining buffer (BCTP/NTB; Diaclone, Besançon, F), plates were dried and measured using an ImmunoSpot ${ }^{B}$ Plate Reader and analyzed with the CTL ImmunoSpot ${ }^{\circledR}$ software (both from Cellular Technology Ltd., Cleveland, USA). The frequency of allo-reactive $\mathrm{T}$ cells was determined as the ratio of the number of spots to the number of responder cells.

\section{Statistical analysis}

Data are expressed as mean values per group \pm SEM. Statistical analysis was performed by means of the non-parametric MannWhitney $U$-test for unpaired data using the software SPSS. Differences between groups were considered significant when $* P<0.05$ and $* * P<0.01$

\section{Acknowledgments}

We would like to thank Mrs Winkler (Max-Delbrück Center for Molecular Medicine, Berlin, D) for her technical assistance in regard to the RESC culture and Elke Effenberger and Sabine Brösel for their excellent technical support. We gratefully acknowledge Dr Ana-Luisa Pina (Berlin-Brandenburg Center for Regenerative Therapies, BCRT) for her critical revision of the manuscript. The project was supported by the BCRT (BMBF funded) and a Charité grant.

\section{References}

1 Fandrich F, Lin X, Chai GX, et al. Preimplantation-stage stem cells induce long-term allogeneic graft acceptance without supplementary host conditioning. Nat Med 2002; 8:171-178.

2 Li L, Baroja ML, Majumdar A, et al. Human embryonic stem cells possess immune-privileged properties. Stem Cells 2004; 22:448-456.

3 Bonde S, Zavazava N. Immunogenicity and engraftment of mouse embryonic stem cells in allogeneic recipients. Stem Cells 2006; 24:2192-2201.

4 Kofidis T, deBruin JL, Tanaka M, et al. They are not stealthy in the heart: embryonic stem cells trigger cell infiltration, humoral and T-lymphocyte-based host immune response. Eur J Cardiothorac Surg 2005; 28:461-466.

5 Swijnenburg RJ, Tanaka M, Vogel H, et al. Embryonic stem cell immunogenicity increases upon differentiation after transplantation into ischemic myocardium. Circulation 2005; 112 Suppl:I166-I172.

6 Pettigrew GJ, Bolton EM, Bradley JA. Alloantigen recognition pathways and transplant tolerance.Transplant Proc 2001; 33:3811-3813.

7 Rogers NJ, Lechler RI. Allorecognition. Am J Transplant 2001; 1:97-102. 
8 Choo JK, Seebach JD, Nickeleit V, et al. Species differences in the expression of major histocompatibility complex class II antigens on coronary artery endothelium: implications for cellmediated xenoreactivity. Transplantation 1997; 64:1315-1322.

9 Pober JS. Immunobiology of human vascular endothelium. Immunol Res 1999; 19:225-232.

10 Collins T, Korman AJ, Wake CT, et al. Immune interferon activates multiple class II major histocompatibility complex genes and the associated invariant chain gene in human endothelial cells and dermal fibroblasts. Proc Natl Acad Sci USA 1984; 81:4917-4921.

11 Hidalgo LG, Halloran PF. Role of IFN-gamma in allograft rejection. Crit Rev Immunol 2002; 22:317-349.

12 Vos IH, Briscoe DM. Endothelial injury: cause and effect of alloimmune inflammation. Transplant Infect Dis 2002; 4:152159.

13 Ferry B, Halttunen J, Leszczynski D, Schellekens H, vd Meide $\mathrm{PH}$, Hayry P. Impact of class II major histocompatibility complex antigen expression on the immunogenic potential of isolated rat vascular endothelial cells. Transplantation 1987; 44:499-503.

14 Ruhnke M, Ungefroren H, Zehle G, Bader M, Kremer B, Fandrich F. Long-term culture and differentiation of rat embryonic stem cell-like cells into neuronal, glial, endothelial, and hepatic lineages. Stem Cells 2003; 21:428-436.

15 Vassilieva S, Guan K, Pich U, Wobus AM. Establishment of SSEA-1- and Oct-4-expressing rat embryonic stem-like cell lines and effects of cytokines of the IL- 6 family on clonal growth. Exp Cell Res 2000; 258:361-373.

16 Wobus AM, Boheler KR. Embryonic stem cells: prospects for developmental biology and cell therapy. Physiol Rev 2005; 85:635-678.

17 Prelle K, Vassiliev IM, Vassilieva SG, Wolf E, Wobus AM. Establishment of pluripotent cell lines from vertebrate species-present status and future prospects. Cells Tissues Organs 1999; 165:220-236.

18 Bordenave L, Fernandez P, Remy-Zolghadri M, Villars S, Daculsi R, Midy D. In vitro endothelialized ePTFE prostheses: clinical update 20 years after the first realization. Clin Hemorheol Microcirc 2005; 33:227-234.

19 Benichou G, Valujskikh A, Heeger PS. Contributions of direct and indirect $\mathrm{T}$ cell alloreactivity during allograft rejection in mice. J Immunol 1999; 162:352-358.
20 Jiang S, Herrera O, Lechler RI. New spectrum of allorecognition pathways: implications for graft rejection and transplantation tolerance. Curr Opin Immunol 2004; 16:550-557.

21 Dye JF, Jablenska R, Donnelly JL, et al. Phenotype of the endothelium in the human term placenta. Placenta 2001; 22:3243.

22 Drukker M, Benvenisty N. The immunogenicity of human embryonic stem-derived cells. Trends Biotechnol 2004; 22:136141.

23 Drukker M, Katz G, Urbach A, et al. Characterization of the expression of MHC proteins in human embryonic stem cells. Proc Natl Acad Sci USA 2002; 99:9864-9869.

24 Drukker M, Katchman H, Katz G, et al. Human embryonic stem cells and their differentiated derivatives are less susceptible to immune rejection than adult cells. Stem Cells 2006; 24:221-229.

25 Holtz R, Choi JC, Petroff MG, Piskurich JF, Murphy SP. Class II transactivator (CIITA) promoter methylation does not correlate with silencing of CIITA transcription in trophoblasts. Biol Reprod 2003; 69:915-924.

26 Miller DM, Rahill BM, Boss JM, et al. Human cytomegalovirus inhibits major histocompatibility complex class II expression by disruption of the Jak/Stat pathway. J Exp Med 1998; 187:675-683.

27 Chang $\mathrm{CH}$, Flavell RA. Class II transactivator regulates the expression of multiple genes involved in antigen presentation. J Exp Med 1995; 181:765-767.

28 Sims TN, Afrouzian M, Urmson J, Zhu LF, Halloran PF. The role of the class II transactivator (CIITA) in MHC class I and II regulation and graft rejection in kidney. Am J Transplant 2001; 1:211-221.

29 Yun S, Rose ML, Fabre JW. The induction of major histocompatibility complex class II expression is sufficient for the direct activation of human CD4+ T cells by porcine vascular endothelial cells. Transplantation 2000; 69:940-944.

30 Liu H, Kemeny DM, Heng BC, Ouyang HW, Melendez AJ, Cao T. The immunogenicity and immunomodulatory function of osteogenic cells differentiated from mesenchymal stem cells. J Immunol 2006; 176:2864-2871.

31 Nicosia RF, Villaschi S, Smith M. Isolation and characterization of vasoformative endothelial cells from the rat aorta. In Vitro Cell Dev Biol Anim 1994; 30A:394-399. 